from increased warmth, the vessels on the surface were relaxed. A comparison of the pulsations of each trace points to the greater rapidity of the circulation under influences which facilitate the passage of the blood.

The force of the pulse is indicated by the height of the pulsations. The greater the elevation of the lever, the greater the energy of the pulse-beat; and we may say that, in many cases, the strength of the ventricular contraction is expressed by the force of the pulse. This law, however, has many exceptions; and we find that the altitude of the pulsetrace depends on several other conditions.

I. The volume of the artery influences much the amplitude of the trace. This can be well seen in traces collected from old persons. In senile changes, the volume of the vessels is increased considerably, and the trace betrays great fulness. Marey believes this to be due, not solely to the hypertrophy of the ventricle which exists in the old, but also to the dilatation of the artery. (Vide trace, Fig. 5.)

Ir. The state of arterial tension modifies greatly the force of the pulse; and, as the tension is dependent on the state of the capillary circulation, it may be said that in most cases " the force of the pulse is not in relation with the energy of the ventricular systole, but that it is regulated by the state of the circulation in the ultimate ramifications of the vascular system." (Marey, op . cit., p. 235.) By means of the manometer, in a great number of experiments, this law has been found to hold good; a feeble state of arterial tension giving to the finger and the instrument the sensation of increased amplitude. Marey, by means of the following diagrams (Figs. 10 and 11), illustrates this very well.

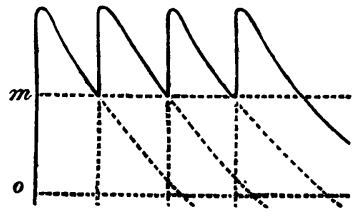

Fig. 10.

The first shows the form of pulsation in a state of feeble tension; the second, under a state of strong tension. The difference in the amplitude of the traces is very distinct. In the state of feeble tension, or easy passage of the blood onwards, the lever falls quickly to the point of least tension, and is ele-

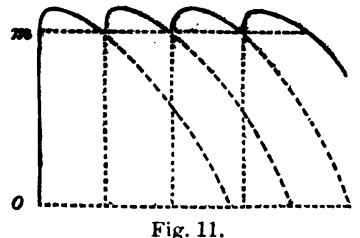

Fig. 11.

vated considerably at each pulsation. In the case of difficult passage of the blood through the capillaries, and consequently of great arterial tension, the lever descends alowly by a hine convex upwards; and, long before it has resebed a minimum tension equal to that in the former case, the lever is raiged alightly by the next pulsation. While the lines of the maxima of arterial tension are the same in both cases, the lines of the minima are very different. On this depends the amplitude of the pulse-trace.

III. The duration of the interval which separates the pulsations ias also a distinct infinence on the rmplitiode of the trace. This is due to the fact that, during a long interval, the blood flowing continually onward lessens the pressure in the ves- sels, and thus favours the greater amplitude of the next pulation. This is well seen in a trace (Fig. $C$ 12) taken from a patient of mine in the Queen's Hospital.

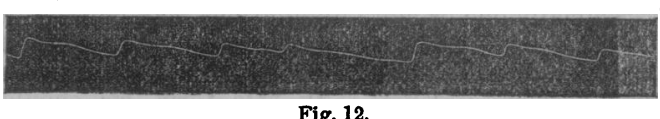

The condition of the vessel itself, as to permeabi-n lity below the point observed, influences the force of ${ }^{\text {s }}$ the pulsation by altering the pressure within the $\overrightarrow{0}$ artery. Marey has also pointed out that in somecases, where the pulse-beat is almost imperceptible $\vec{\omega}$ to the finger, the sphygmograph records a consider-o able amplitude of trace; and vice versâ. I have beeno struck with this peculiarity in several instances: $?$. and the explanation appears to be, that we perceiveehanges in the artery more distinctly in proportionis as they occur more suddenly. The cases in which $N$ the pulse has been almost imperceptible to the touch, but has nevertheless yielded a distinct trace. have been associated with a very slow distension of the ressels.

The foregoing remarks, based upon the work of the French author, will, I trust, by pointing out the $V$ meaning of the many forms of pulse-trace, and by setting before us the causes to which they are due, enable us to understand more clearly the changes exhibited by the arterial pulse-form in disease. The $\rightarrow$ remainder of the paper $I$ shall, with the permissiono of the Bociety, devote to a consideration of some fews of the many pathological conditions in the detection of which the use of the sphygmograph renders us in-o valuable aid.

[To be contixued.]

\section{ON THE USE OF THE THERMOMETER $\stackrel{\complement}{\rightrightarrows}$ AS A GUIDE IN THE DIAGNOSIS OF PYREXIAL DISEASES.}

By F. W. Gibson, B.A., M.D.London, late House Surgeon of the Taunton and Somerset Hospital, Taunton.

[Concluded from page 251.]

Typical Range of Temperature in different Diseases.

A mowhores of the range of tempersture peontiari to each disease enables the obeerver, when ho per-3 ceives any marked departure from the type, to pro-o nounce either that his diagnosis is rrong, and that the disease is not what it appeared to be, or that $N$ some secondary lesion has supervened.

For example, the typical range or temperature in을 typhoid (Peyerian) fever, as established by Wunder- $N$ lich, is as follows.

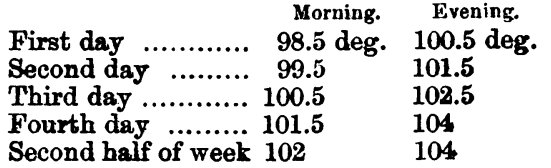

At the commencement of the second week, in mild $\square$ cases, the temperature begins to decrease; for, al-o though the evening temperature may be 104 deg., $\mathbb{D}$ the morning is only $102 \mathrm{deg}$. In severe cases, on $\frac{?}{\mathrm{D}}$ the other hand, the morning temperature is above 103 deg.; the evening above $104.5 \mathrm{deg}$., and mayo reach $106 \mathrm{deg}$. In the third week, remarkable vacillations of four to six degrees between the morning ando evening temperature occur. The fever terminates 
by a gradual defervescence (lysis), not by a sudden crisis, as in typhus. In mild cases, the fever lasts from three to four weeks ; in severe, from four and a half to ten weeks.

Typhoid fever is not present, if, on the evening of the first or second day of the disease, the tempersture be $104 \mathrm{deg}$; if the evening temperature do not rise to 103.3 deg. between the fourth and sixth days; if, in the second half of the first week, the evening temperature decrease considerably; lastly, if, between the eighth and eleventh days, the temperature be below $103 \mathrm{deg}$.

As an illustration of the truth of the first proposition, I cite the succeeding case. 1 woman, in a ward of the hospital in which there were two or three cases of typhoid fever, had one evening an attack of rigors. Her temperature was $102 \mathrm{deg}$; ; on the morning of the next day, $103 \mathrm{deg}$; in the evening, 105 deg. The symptoms in all other respects resembled those of incipient typhoid. The woman was mueh alarmed, for a patient had just died of typhoid fever. The existence of a temperature of $105 \mathrm{deg}$. in the evening of the second day enabled her to be assured that she was not threatened with that disease. This prognosis was proved to be correct by the symptoms developing shortly into those of erysipelas.

The next case is an example of the value of the second and third propositions. A girl, aged 21, was admitted on January 15th, 1866, with symptoms of impending typhoid. The attack began with rigors on the evening of the 11th. She had great abdominal tenderness, a hot dry skin, pains in the limbs, muscular tremors, and one or two suspicions specks on the skin of the chest. Nevertheless, because the temperature did not on any evening rise to $103 \mathrm{deg}$., and a marked decrease of temperature occurred on the evening of the 18th, the disease was pronounced not to be typhoid. On the 20th, she was quite well.

The next case illustrates the last proposition. A man, aged 40 , living in a locality in which typhoid had lately been rife, who had had rigors, followed by diarrhcea and pains in the limbs, a week previous to his admission, was found to have a typhoid tongue, frontal headache, diarrhœa, and numerous specks on his abdomen and chest, very closely resembling the eruption peculiar to typhoid ferer. On the tenth evening of the disease, his temperature was 101.8 deg. This temperatare forbad the diagnosis of typhoid. In three days after, the man was well.

The effect of hæmorrhage in reducing the temperature was seen in the case of a woman with typhoid fever, whose temperature fell suddenly from a high degree to $98 \mathrm{deg}$. This led to an inquiry after intestinal hæmorrhage; and it was discovered that she had lost a considerable quantity of blood from the intestines, which accident the nurse omitted to mention until she was questioned.

Range of Temperature in Traumatic Erysipelas. In all of the cases of traumatic erysipelas which $I$ have observed thermometrically, the attack began with a fit of rigors more or less severe. The fastigium, or highest point of the temperature, was attained in a short time. The fall, or defervescence of temperature, nearly coincident with the decline of the eruption, and accompanied in the majority of the cases by sweating, was rapid. In some of the cases, relapse occurred.

If the temperature remain high for any consider. able number of hours after the disappearance of the eruption, some secondary complication-abscess, pneumonia, pleurisy, pericarditis, or pyæmia-has undoubtedly supervened.

CASE I. On February 8th, 1866, the operation for ectropium was performed on a man aged 33. At 3.30
P.M. on Feb. 9th, he had an attack of rigors. At 4.10 P.M., his temperature was 103.6 deg.; pulse 120 ; respirations 20 . There was slight redness round the wound. At 6 P.M., the temperature was 104 deg.; pulse 100 ; respirations 24 . He was then sweating. Feb. 10th, 9 A.M. Temperature 100.8 deg.; pulse 84; respirations 20 . The erysipelas was much less. At 6 P.M., the temperature was 102 deg.; pulse 100. Feb. 11th, 9 A.m. Temperature 100.8 deg.; pulee 84; respirations 24. The erysipelas was gone. 6 P.M. Temperature 101 deg.; pulse 84; respirations 24. Feb. 12th, A.M. Temperature 99.8 deg.; pulse 72; respirations 20 . There was no relapse.

CAsE II. This case was one of erysipelas of the leg in a man aged 40 . On Feb. 16th, 1866, he had rigors at 1 P.M. At 5.50 P.M., the temperature was $103.8 \mathrm{deg}$; pulse 68 ; respirations 20 . There was a slight erysipelatous blush on the leg, and angina fancium. 8.46 P.M. Temperature $102.6 \mathrm{deg}$.; pulse 80; respirations 20 . He was then sweating. Feb.17th, 12.30 A.M. Temperature 101.2 deg. ; pulse 64 ; respirations 20. 6 A.m. Temperature $99.6 \mathrm{deg}$.; pulse 48 ; respirations 20. 9.30 A.m. Temperature 99 deg.; pulse 64 ; respirations 24 . The erysipelas had nearly disappeared. 6. P.x. Temperature $99 \mathrm{deg}$. ; pulse 68; respirations 24. Feb. 18th, 6 P.x. Temperatare 99.8 deg.; pulse 58; respirations 24. Feb. 19th. The patient had a fit of rigors at 12 A.M., denoting another attack of erysipelas. At 6 P.M., the temperature was 103.4 deg.; pulse 80 ; respirations 24 .

The usefalness of the thermometer in the diagnosis of pyrexia is well shown in this case; for, although the temperature on February 19th, P.M., was 108.4 deg., Jet the pulse was only 80 , the respirations but 24, and the skin was objectively cool.

CASE III. A woman, aged 49, was operated on for cancer of the breast on January 22nd, 1866. The wound being nearly healed, she had, at 4 A.M. on February 12th, an attack of rigors. At 9 A.M., the temperature was $103 \mathrm{deg}$; pulse 120 ; and there was an erysipelatous blush around the wound. At 10.30 A.M., she had another attack of rigors. 11.35 A.M. Temporature $105.4 \mathrm{deg}$.; pulse 120 ; respirations 24. 6 P.M. Temperature $105 \mathrm{deg}$. ; pulse 108 ; respirations 24. Feb. 13th, 9 A.M. Temperature 105. deg.; pulse 120 ; respirations 20 . The erysipelas was extending. 6 P.M. Temperature $104.6 \mathrm{deg}$; pulse 108 ; respiratiions 24. Feb. 14th, 6 P.x. Temperature $104 \mathrm{deg}$. pulse 120 ; respirations 24 . The erysipelas was subsiding. Feb. 16th, 6 P.M. Temperature 104 deg.; pulse 120; respirations 24. The erysipelas was nearly gone. She had no ccugh; no pain in the chest. There was dulness at the base of the left lung; no crepitation. The respiration-sound was rather feeble. Feb: 17th, 9 A.m. Temperature 103 deg.; pulse 120 ; respirations 28 . There was dulness over the left lung as high as the inferior angle of the scapula; no crepitation.

In this case, the fact that the temperature remained, on February 16th, at so high a degree after the subsidence of the erysipelatous eruption, led to the diagnosis of the secondary complication; for no other signs of the lung-disesse save the continuance of the high temperature existed. There was no cough; no pain in the chest; the respirations were not accelerated; the pulse was feeble, so that its rapidity might have been due to weakness; and, lastly, the skin felt abnormally cool.

Erratum. In Dr. Gibson's paper published in last week's JouRNAL, page 249 , column 2, line 28 from bottom, for "covered near the bulb", read "curved near the bulb". 